\title{
Erbium emission in MOS light emitting devices: from energy transfer to direct impact excitation
}

\author{
J M Ramírez ${ }^{1}$, F Ferrarese Lupi ${ }^{1}$, O Jambois ${ }^{1}$, Y. Berencén ${ }^{1}$, D Navarro-Urrios ${ }^{1,4}$, A Anopchenko ${ }^{2}$, A \\ Marconi $^{2}$, N Prtljaga, A Tengattini ${ }^{2}$, L Pavesi ${ }^{2}$, Jean-Phillippe Colonna ${ }^{3}$, J M Fedeli ${ }^{3}$ and B Garrido ${ }^{1}$.
${ }^{1}$ Departament d'Electrònica, Universitat de Barcelona, Carrer Martì i Franquès 1, Barcelona 08028, Spain. ${ }^{2}$ Nanoscience Laboratory, Department of Physics, University of Trento, Via Sommarive 14, Povo (Trento) 38123, Italy ${ }^{3}$ CEA, Léti, Minatec campus 17 rue des Martyrs, 38054 Grenoble cedex 9, France. \\ ${ }^{4}$ Catalan Institute of Nanotechnology (CIN2-CSIC), Campus UAB, edifice CM3, 08193 Bellaterra, Spain. \\ jmramirez@el.ub.es
}

\begin{abstract}
The electroluminescence (EL) at $1.54 \mu \mathrm{m}$ of metal-oxide-semiconductor (MOS) devices with $\mathrm{Er}^{3+}$ ions embedded in the silicon-rich silicon oxide (SRSO) layer has been investigated under different polarization conditions and compared with that of erbium doped $\mathrm{SiO}_{2}$ layers. EL time-resolved measurements allowed us to distinguish between two different excitation mechanisms responsible for the $\mathrm{Er}^{3+}$ emission under an alternate pulsed voltage signal (APV). Energy transfer from silicon nanoclusters (Si-ncs) to $\mathrm{Er}^{3+}$ is clearly observed at low-field APV excitation. We demonstrate that sequential electron and hole injection at the edges of the pulses create excited states in Si-ncs which upon recombination transfer their energy to $\mathrm{Er}^{3+}$ ions. On the contrary, direct impact excitation of $\mathrm{Er}^{3+}$ by hot injected carriers starts at Fowler-Nordheim injection threshold (above $5 \mathrm{MV} / \mathrm{cm}$ ) and dominates for high-field APV excitation.
\end{abstract}

Keywords: Silicon nanocrystals, electroluminescence, erbium, direct impact, energy transfer.

\section{Introduction}

The realization of an efficient and integrated silicon based light emitter is considered as one of the key points for silicon photonics to achieve monolithic electronic and photonic functional integration in the same chip. In particular, $\mathrm{Er}^{3+}$ doped materials have been thoroughly studied, as the radiative $4 \mathrm{f}$ shell intraband transition provides efficient emission at telecom wavelengths $(1.54 \mu \mathrm{m})[1-3]$. 
Several works have attributed the silicon-rich silicon oxide (SRSO) system as the most promising host for $\mathrm{Er}^{3+}$ ions $[4,5,6]$. When an as-deposited SRSO undergoes an annealing at high temperature, the silicon in excess segregates into a nanophase composed of nanoclusters and/or nanocrystals depending on the annealing conditions. The optimized environment of the erbium ions inside the silicon oxide matrix and the contribution of silicon nanostructures to $\mathrm{Er}^{3+}$ emission results in an enhancement and a spectral broadening of the effective excitation crosssection at $1.54 \mu \mathrm{m}$ under optical pumping [5-8].

Nonetheless, under electrical pumping, the coupling between silicon nanoclusters or nanocrystals (Si-ncs) and $\mathrm{Er}^{3+}$ ions is still controversial. Some authors have reported an enhancement of the $\mathrm{Er}^{3+}$ electroluminescence (EL) in SRSO at low voltages [9, 10]. Others reported a diminution of the EL signal for increasing Si excess and postulated leaking currents through $\mathrm{Si}$-ncs paths and/or less energetic injected carriers -in comparison with pure $\mathrm{SiO}_{2^{-}}$as the main reason for decreasing efficiency [11].

The visible/near infrared emission of Si-ncs under different polarization conditions i.e. direct current (DC) or alternate pulsed voltage regime, has been widely studied. In APV conditions, EL at low voltages is greatly enhanced due to the sequential injection of electron and holes into $\mathrm{Si}$-ncs at the edges of the pulses and the following radiate recombination $[12,13]$. However, the behaviour of $\mathrm{Er}^{3+}$ emission under APV excitation has not been studied in detail. If indirect excitation of $\mathrm{Er}^{3+}$ ions (through Si-ncs) would efficiently take place for electrical excitation, APV polarization could bring about significant improvement in the performance of $\mathrm{Er}^{3+}$ MOSLEDs (MOS capacitor or transistor acting as light emitting device).

In this work, we have fabricated Si-based MOSLEDs containing $\mathrm{Er}^{3+}$ ions embedded in either stoichiometric $\mathrm{SiO}_{2}$ or SRSO layers. We have characterized the electrical and optical response when polarizing MOSLEDs under DC or APV signals. Results show two different EL excitation mechanisms for the coupled system with Si-ncs and $\mathrm{Er}^{3+}:$ i) Indirect excitation: cold carriers excite Si-ncs by sequential injection and the energy is transferred to $\mathrm{Er}^{3+}$ and ii) Direct excitation: $\mathrm{Er}^{3+}$ is directly energized by hot energetic carriers through impact excitation. Moreover, we demonstrate the existence of a narrow voltage window, where the $\mathrm{Er}^{3+}$ excitation 
mechanism changes from energy transfer to direct impact excitation. Finally, a fast EL overshoot has been observed at high APV voltage signals, attributed to the emission of high stressed defects in the host matrix.

\section{Sample fabrication and experimental setup}

Two series of MOS devices were fabricated using standard complementary metal-oxidesemiconductor (CMOS) techniques, where the oxide layer has been replaced either by a SRSO or by a dry thermal silicon oxide with the same $\mathrm{Er}^{3+}$ implantation. In the following, all the devices with a SRSO layer will be labeled as 'device 1', while those containing a thermal silicon oxide will be labeled as 'device 2'.

Concerning device 1 a $50 \mathrm{~nm}$ thick substoichiometric $\mathrm{SiO}_{\mathrm{x}}(\mathrm{x}<2)$ film was deposited on a low resistivity p-type Si substrate by low pressure chemical vapor deposition (LPCVD). The nominal value of silicon excess in the matrix was $12 \%$, allowing the Si-nc formation after annealing at $900{ }^{\circ} \mathrm{C}$ during $1 \mathrm{~h}$. The same annealing treatment was applied to grow the thermal oxide in device 2.

After the annealing treatment both films were implanted with $\mathrm{Er}^{3+}$ ions with a dose of $10^{15}$ at $/ \mathrm{cm}^{2}$ at $20 \mathrm{KeV}$ of energy. Finally, a post-implantation annealing was performed in order to activate $\mathrm{Er}^{3+}$ ions and cure the matrix from the implantation. Further details concerning $\mathrm{Er}^{3+}$ implantation profiles, PL, XPS and SIMS measurements can be found in ref. 14.

In addition, $100 \mathrm{~nm}$ thick poly-crystalline silicon of n-type doped layer $\left(1 \times 10^{20} \mathrm{at} / \mathrm{cm}^{3}\right)$ was deposited on top and used as optically semi-transparent gate electrode. 100x100 $\mu^{2}$ aluminum pads were photolitographically defined on the devices to facilitate the electrical polarization and light extraction. The emission area is composed by a square of $0.09 \mathrm{~mm}^{2}$ area. The crosssection of the devices can be observed on the inset of figure 1a.

A semiconductor device analyzer (Agilent B1500A) and a probe station (Cascade Microtech Summit 1100) were used for current-voltage (I-V) measurements. APV excitation under a square voltage signal at low frequencies was performed replacing the semiconductor device analyzer by a pulse generator (Agilent $8114 \mathrm{~A})$. 
EL signals were analyzed by an Acton $2300 \mathrm{i}$ grating spectrometer and detected by a cryogenically cooled PI spec-10-100B/LN charge-coupled device or a photomultiplier tube (H10330-25). A digital oscilloscope was finally used for data collection with a temporal resolution of $1 \mu \mathrm{s}$.

The micro photoluminescence ( $\mu \mathrm{PL}$ ) measurements were performed in a standard $\mu \mathrm{PL}$ setup in a 45 degrees configuration. The PL signal of the samples was studied by using two different lines of an Argon LASER (488 nm and $476 \mathrm{~nm}$ ) as a pump source. The $488 \mathrm{~nm}$ line is resonant with the ${ }^{4} \mathrm{I}_{15 / 2} \rightarrow{ }^{4} \mathrm{~F}_{7 / 2}$ transition of the $\mathrm{Er}^{3+}$ ions while the $476 \mathrm{~nm}$ line is non-resonant with an $\mathrm{Er}^{3+}$ transition and only excites the Si-ncs, which have slightly higher excitation cross section at this wavelength than at $488 \mathrm{~nm}$.

The shape of the pumping spot on the sample is ellipsoidal, with an area of $6 \times 10^{-5} \mathrm{~mm}^{2}$, which is small enough to ensure the excitation of one single device.

A short working distance objective was used to collect the PL emission and to focus it in a monochromator with focal length of $750 \mathrm{~mm}$ and a spectral resolution of $0.03 \mathrm{~nm}$, coupled to the same photomultiplier tube described above.

\section{Results and discussion}

\subsection{Electro-optical characterization}

The electrical excitation in DC was done under accumulation condition, i.e. applying negative voltages over the gate electrode with the substrate grounded (see figure 1b). This configuration is more convenient as it provides majority of carriers both from the gate electrode (electrons) and from the substrate (holes). Quasi-static I(V) characteristics of devices at room temperature are shown in figure $1(20 \mathrm{mV} / \mathrm{s})$. A difference of $17 \mathrm{~V}$ between the SRSO layer (device 1) and the pure $\mathrm{SiO}_{2}$ (device 2) is observed in the threshold voltage for conduction $\left(\mathrm{V}_{\mathrm{TH}}\right)$, defined arbitrarily as the voltage required to obtain $1 \mathrm{nA}$ of gate current. It is worth to note that a homogeneity study was performed on each wafer obtaining around $95 \%$ of reproducibility in the electro-optical characteristics. 
a)

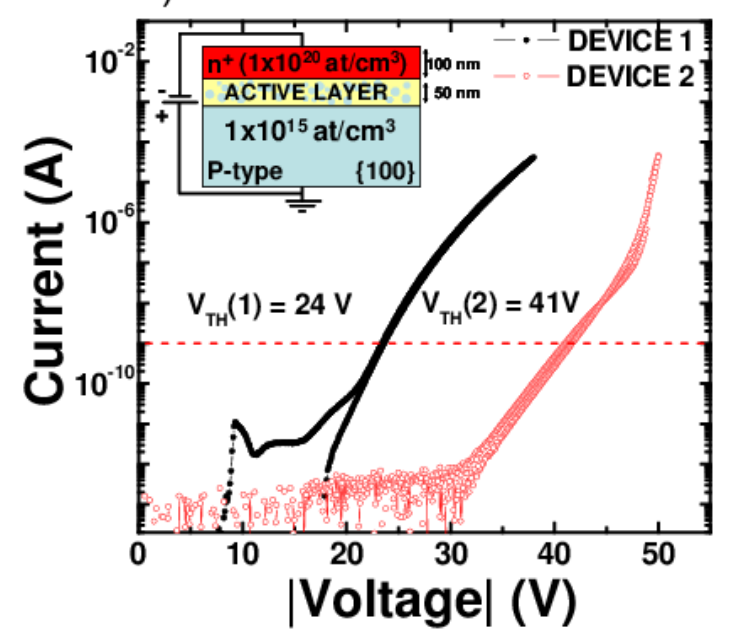

b)

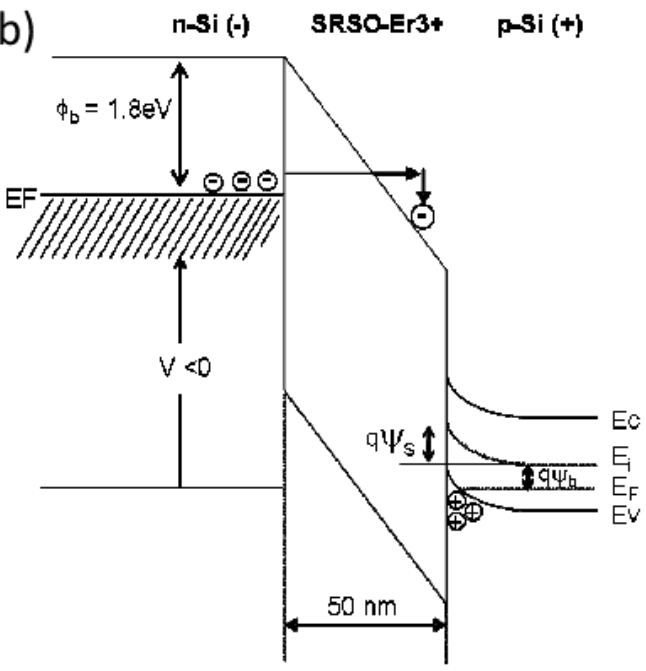

Figure 1: (a) Quasi-static I(V) characteristics of devices. (b) Energy band diagram of the structure under high field condition for device 1.

It is thus clear that Si-ncs are responsible for an increased conductivity in the SRSO with respect to pure $\mathrm{SiO}_{2}$. By a detailed fitting procedure, it has been determined that the dominant conduction is injection limited and proceeds for both $\mathrm{SRSO}$ and $\mathrm{SiO}_{2}$ (after a threshold voltage) by Fowler-Nordheim tunnel injection of hot electrons coming from the gate electrode [15]:

$$
J=\frac{\mathrm{q}^{3} \mathrm{E}^{2}}{8 \pi \mathrm{h} \phi_{\mathrm{b}}} \exp \left(-\frac{4 \sqrt{2 \mathrm{~m}_{\mathrm{ox}}^{*}\left(\mathrm{q} \phi_{\mathrm{b}}\right)^{3}}}{3 \hbar \mathrm{qE}}\right)
$$

Where $\mathrm{m}_{\mathrm{ox}}{ }^{*}$ is the effective mass of electrons in the conduction band, E the electric field applied, $\phi_{\mathrm{b}}$ the potential barrier height, q the single electron charge and $\mathrm{h}(\hbar)$ the (reduced) Planck's constant.

The agreement with the F-N conduction law suggests that both Si-ncs and $\mathrm{Er}^{3+}$ do not create a large number of trapping defects in the oxide as, if this was the case, conduction would proceed by a Poole Frenkel type mechanism [16].

Then, assuming that the erbium implantation does not lower down the $\mathrm{Si}_{-} \mathrm{SiO}_{2}$ barrier height, the effective mass for the $\mathrm{Er}^{3+}$ doped $\mathrm{SiO}_{2}$ can be extracted from the experimental $\mathrm{I}(\mathrm{V})$ curves. Using equation 1 and the accepted value for the $\mathrm{Si}_{-} \mathrm{SiO}_{2}$ barrier height at the interface, i.e. 3.15 $\mathrm{eV}[17,18]$, an effective mass of $\mathrm{m}_{\mathrm{ox}}{ }^{*}=0.4 \mathrm{~m}_{\mathrm{o}}\left(\mathrm{m}_{\mathrm{o}}\right.$ the electron mass) was found, in agreement with the ones previously reported in $\mathrm{Si}_{-} \mathrm{SiO}_{2}$ systems [19]. On the other hand, doing the same 
study for the $\mathrm{Er}^{3+}$ doped SRSO layer, we obtained a barrier height of $1.6 \mathrm{eV}$ using an effective mass of $0.4 \mathrm{~m}_{0}$, or $1.8 \mathrm{eV}$ using $\mathrm{m}_{\mathrm{ox}}{ }^{*}=0.5 \mathrm{~m}_{\mathrm{o}}$ as taken by various authors $[18,20]$. In both cases, a reduction of the injection barrier height is obtained when Si-ncs are introduced.

A thorough study on the evolution of the EL at $1.54 \mu \mathrm{m}$ as a function of the applied DC voltage is reported in figure 2. The voltages were swept from low to just below the breakdown voltage, integrating the whole EL spectrum at each voltage value (see inset of figure 2). However, care should been taken with the highest electric fields applied (above $7 \mathrm{MV} / \mathrm{cm}$ for device 1 and 10 $\mathrm{MV} / \mathrm{cm}$ for device 2), as Si bulk emission at $1.1 \mu \mathrm{m}$ has been observed in the spectra leading to a tail until the $1.5 \mu \mathrm{m}$ region. In this regime, the active layer is not thick enough to cool down the high energetic electrons from the gate, which pass through the layer and impact the silicon bulk directly. Furthermore, at these high electric fields the devices are partially broken due to the high electric stress, providing; i) higher leakage currents for a given voltage; ii) lower EL values for a given current; and iii) poor device reliability. As a consequence, the depletion of the EL(V) characteristic at high voltages can not be considered as a complete saturation of the optically active $\mathrm{Er}^{3+}$ ions, but to a diminution of the electro-optical properties of the devices.

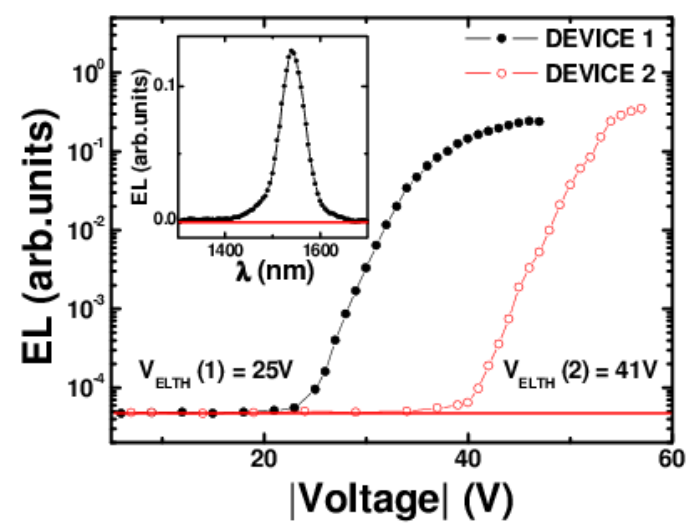

Figure 2: $E L(V)$ characteristics for devices. The inset shows a typical measured spectrum $(\mathrm{Vg}=-40 \mathrm{~V}$ for device 1$)$ 
Therefore, the optimum working conditions were established below these maximum fields. Additionally, the threshold voltage for the electroluminescence $\left(\mathrm{V}_{\mathrm{EL}-\mathrm{TH}}\right)$ can be extracted from figure 2, determining a minimum voltage for light emission of $25 \mathrm{~V}$ in device $1(5 \mathrm{MV} / \mathrm{cm})$, and $41 \mathrm{~V}(8 \mathrm{MV} / \mathrm{cm})$ for device 2 . These values are very similar to the threshold voltage for conduction and allow extracting a minimum gate current to observe EL of around $1 \mathrm{nA}$. From those results, the power efficiency was found to be very similar in both devices with values around $0.01 \%$, pointing out that the emission properties at $1.54 \mu \mathrm{m}$ are not enhanced by the presence of Si-ncs, although the conductivity across the active layer is improved.

In order to study the dynamics of our system in the infrared region, time resolved EL measurements centered at $1.54 \mu \mathrm{m}$ were carried out. The time evolution of the EL signal at negative square voltages is reported on figure $3 \mathrm{a}$ for the $\mathrm{Er}^{3+}:$ SRSO sample. The signal can be modelled by using the rate equations for a nearly-two level system [21, 22],

$$
\frac{\mathrm{dN}_{2}}{\mathrm{dt}}=\sigma \frac{\mathrm{j}}{\mathrm{e}}\left(\mathrm{N}_{\text {total }}-\mathrm{N}_{2}\right)-\frac{\mathrm{N}_{2}}{\tau_{\text {decay }}}, \quad \text { (2) with } \quad \frac{1}{\tau_{\text {decay }}}=\frac{1}{\tau_{\text {rad }}}+\frac{1}{\tau_{\text {non-rad }}}
$$

Where $\sigma$ is the effective excitation cross-section, $\mathrm{j}$ is the incident current density, $\mathrm{e}$ is the single electron charge, $\tau_{\text {decay }}$ is the total lifetime (radiative $\left(\tau_{\text {rad }}\right)$ and non-radiative $\left(\tau_{\text {non-rad }}\right)$ ) and $\mathrm{N}_{\text {total }}$ and $\mathrm{N}_{2}$ are the total implanted and the excited $\mathrm{Er}^{3+}$ ions in the first energy level, respectively. When the electric excitation is turned on, the EL intensity behaves as:

$$
E L_{o n}(t)=E L_{0}\left\{1-\exp \left[-\left(\sigma \frac{j}{e}+\frac{1}{\tau_{\text {decay }}}\right) t\right]\right\}
$$

Being $\mathrm{EL}_{0}$ the electroluminescence in the steady state. The rise time will hence follow the equation:

$$
\frac{1}{\tau_{\text {rise }}}=\frac{1}{\tau_{\text {decay }}}+\frac{j}{e} \sigma
$$

and the expression for the turn off EL:

$$
E L_{o f f}(t)=E L_{0} \exp \left(-\frac{t}{\tau_{\text {decay }}}\right)
$$


Therefore, using equation 4 and equation 6 to determine the time constants $\left(\tau_{\text {rise }}\right.$ and $\left.\tau_{\text {decay }}\right)$, the effective excitation cross-section at $1.54 \mu \mathrm{m}$ was extracted. Figure $3 \mathrm{a}$ shows the fits of the EL signal under a square APV voltage from 0 to $-30 \mathrm{~V}$ at $50 \mathrm{~Hz}$ (figure $3 \mathrm{~b}$ ) with a rise time of $1 \mathrm{~ms}$ and a decay time of $1.2 \mathrm{~ms}$ [22]. An effective excitation cross-section value of $\sigma=(5.6 \pm 1) \times 10^{-14}$ $\mathrm{cm}^{2}$ was determined for the $\mathrm{Er}^{3+}:$ SRSO sample, and $\sigma=(5.8 \pm 1) \times 10^{-14} \mathrm{~cm}^{2}$ for the $\mathrm{Er}^{3+}$ doped $\mathrm{SiO}_{2}$ layer, with a given current density ranging from $1 \mu \mathrm{A} / \mathrm{cm}^{2}$ to $5 \mathrm{~mA} / \mathrm{cm}^{2}$ (figure $3 \mathrm{c}$ ). Then, the resemblance between both values becomes a conclusive proof of the direct excitation of $\mathrm{Er}^{3+}$ ions under DC polarization at high voltages, even when Si-ncs are present in the matrix. Notice that the effective excitation cross-sections presented in the present work are the highest ever reported $[23,24]$. The understanding of these values needs further investigation.
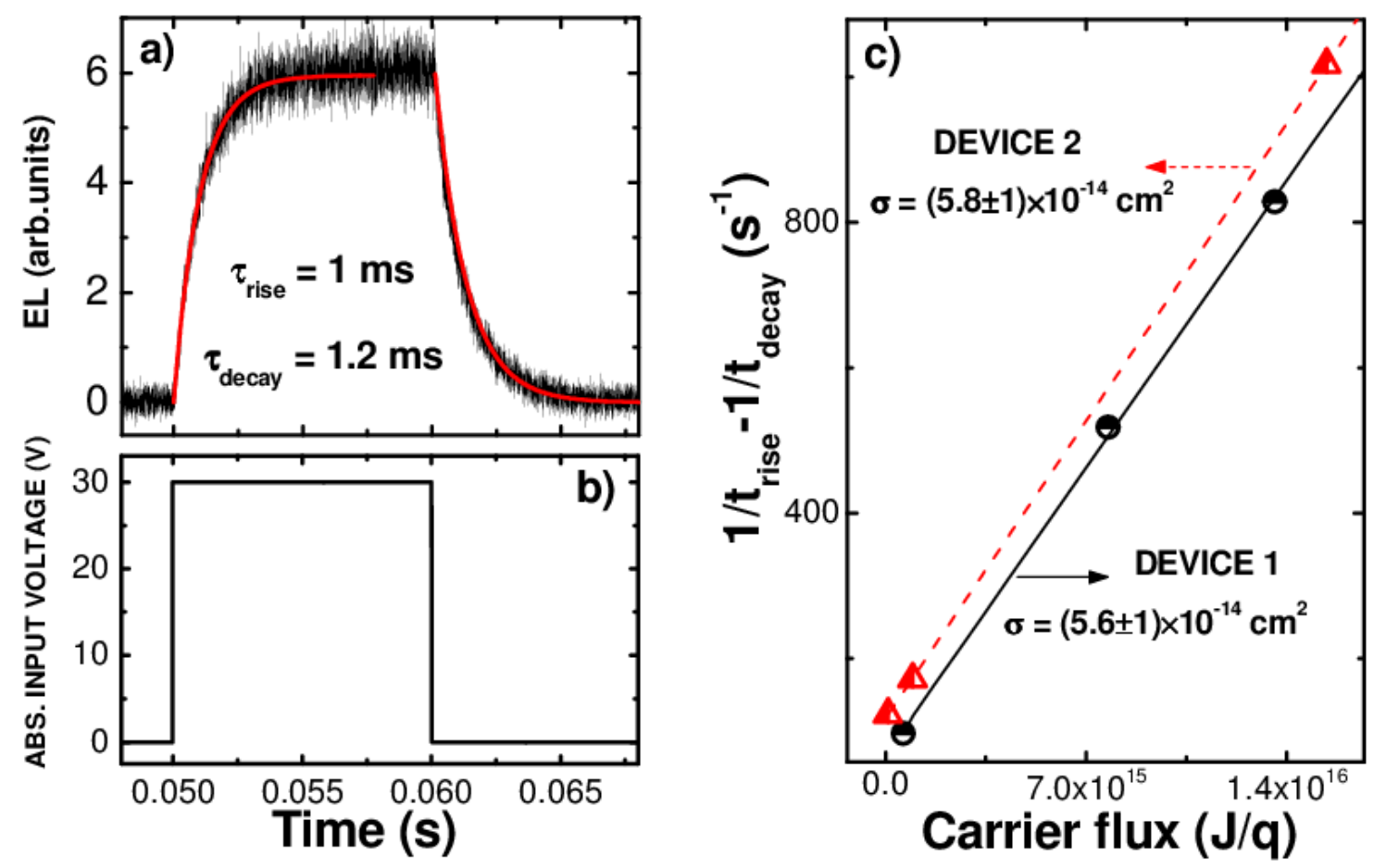

Figure 3: a) EL(t) at $1.54 \mu \mathrm{m}$ of device 1 with the exponential fits of the decay and rise times. b) A negative square voltage (from 0 to $-30 \mathrm{~V}$ ) was used for the excitation. c) Effective excitation cross-section fit at $1.54 \mu \mathrm{m}$ for the $\mathrm{Er}^{3+}$ :SRSO device (solid line, black circles) and for the $\mathrm{Er}^{3+}: \mathrm{SiO}_{2}$ system (dashed line, red triangles). Error bars are smaller than the experimental points shown. 


\subsection{Symmetric alternate pulsed polarization for energy transfer activation}

The transient EL signal was also studied in our devices when polarizing under symmetric square voltage signals (i.e. equal negative and positive voltages relative to zero) at frequencies ranging from $25 \mathrm{~Hz}$ up to $1 \mathrm{KHz}$, which corresponds with the typical lifetime of $\mathrm{Er}^{3+}$ ions (1 ms). Figure 4 shows the EL at $1.54 \mu \mathrm{m}$ for a square voltage switched from $-20 \mathrm{~V}$ to $20 \mathrm{~V}$ at $25 \mathrm{~Hz}$, below the EL threshold voltage in DC. The first thing to point out is that, contrary to the DC case, EL is observed at $1.54 \mu \mathrm{m}$ at this low applied voltage, as shown by the EL transients that appear at each voltage switch (by fixing the monochromator at $1.54 \mu \mathrm{m}$, see figure $4 \mathrm{c}$ ). Decay times were fitted for both peaks, obtaining values of $1.5 \mathrm{~ms}$ (figure $4 \mathrm{a}$ ) and $1.8 \mathrm{~ms}$ respectively (figure $4 \mathrm{~b}$ ). Those values are very close to the decay lifetimes measured in Fig. 3a and Fig. 3b under a negative APV excitation. Moreover, very fast rise times were observed in the EL when the voltage is switched, showing up the characteristic time $(\tau=\mathrm{RC})$ of the setup (about $1 \mu \mathrm{s}$ ).
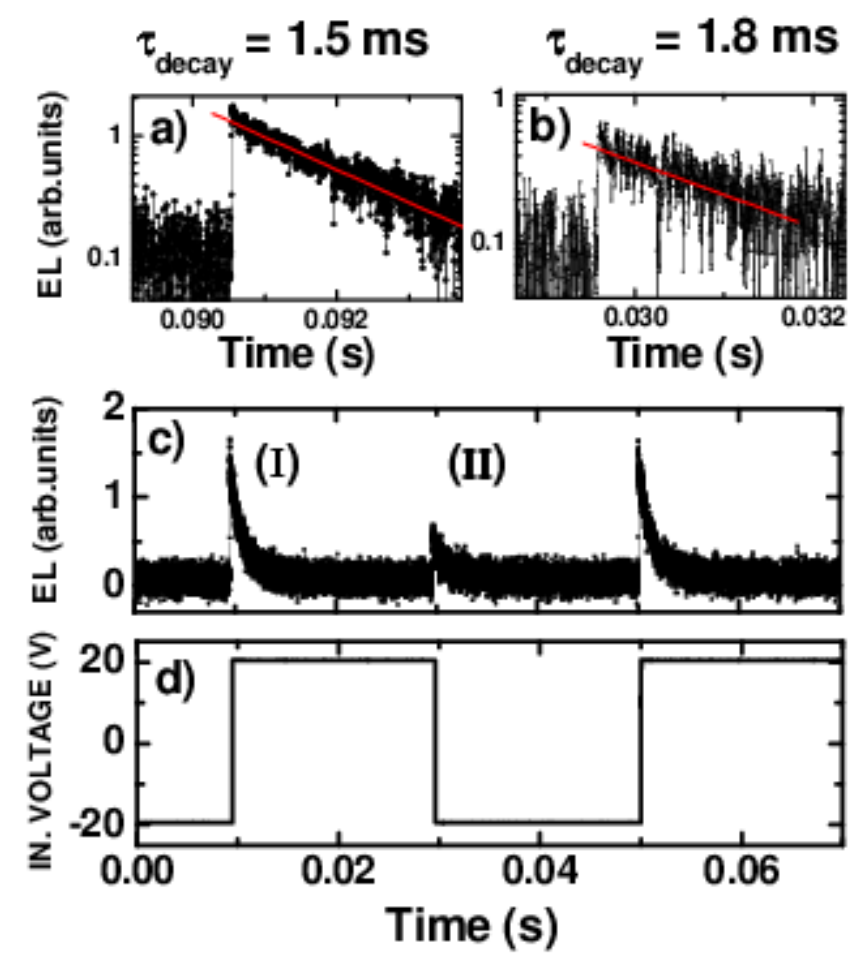

Figure 4: Time-resolved EL measurements (c) performed in device 1 under a square voltage signal (d), with time decay fits for the first (I) and the second (II) EL overshoots (figures a and b, respectively). 
The presence of these two peaks was already reported on the visible region by other authors when working in APV excitation condition with undoped Si-ncs based devices [25], and was ascribed to the sequential injection of holes and electrons into Si-ncs. Also, the difference of intensities between these two peaks can be explained considering that holes have tunneling back times longer than electrons, increasing the probability to create an exciton at the positive semicycle (electrons tunneling towards Si-ncs). Therefore, the observed temporal behavior of the infrared EL (smaller rise time) suggests the influence of Si-ncs on the emission under this polarization, and more concretely their function as sensitizers for $\mathrm{Er}^{3+}$ ions. Moreover, time resolved measurements were also performed in the visible range under the same polarization conditions, showing two EL peaks similar to the ones reported on figure 4c (not shown).

A more in-depth evidence of the indirect excitation of the $\mathrm{Er}^{3+}$ ions for this excitation scheme comes out from the comparison, in device 1, of the EL rise time under both negative (empty squares) and symmetrical (empty circles) square pulse voltage signals (see figure 5). Starting from the assumption that the energy transfer from $\mathrm{Si}$-ncs to $\mathrm{Er}^{3+}$ ions is known to be a very fast process [26], we report a difference of at least $200 \mu$ s in the temporal EL response depending on the polarization scheme (see inset of figure 5). In addition, looking at the rise times, we observe a factor of $\sim 1000$ between them (lower than $1 \mu \mathrm{s}$ in front of $1 \mathrm{~ms}$ ) which suggest very different excitation cross-sections (also a factor of 1000) if equation 5 is assumed. Consequently a correlation between the slower rise time (empty squares) with the direct excitation of $\mathrm{Er}^{3+}$ ions, and the faster (empty circles) with the energy transfer between Si-ncs to $\mathrm{Er}^{3+}$ ions was inferred. 


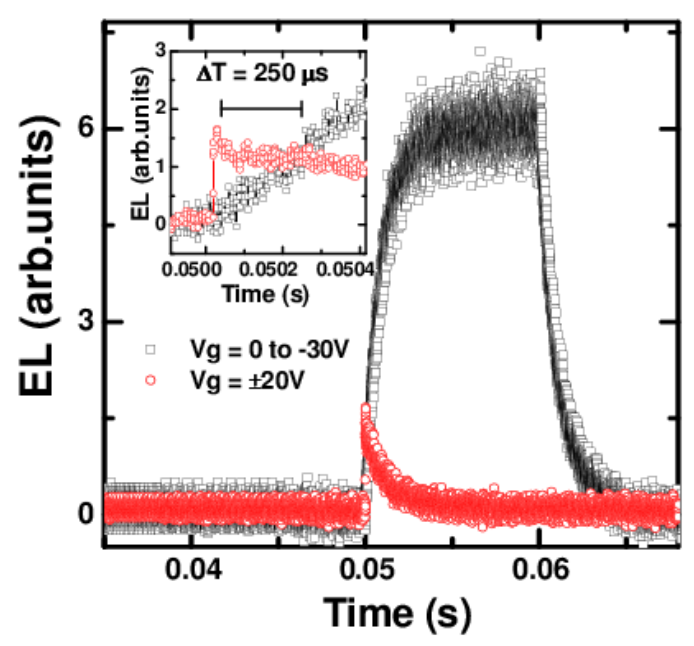

Figure 5: Rise time comparison between the EL obtained under a symmetric AC signal (empty circles) and a negative square signal (empty squares). The inset is a zoom of the rising EL.

Visible and infrared spectra were acquired under different electric polarization conditions, in order to correlate the emission of $\mathrm{Si}$-ncs and $\mathrm{Er}^{3+}$ ions. Figure 6 shows the visible spectra of the EL under both DC and APV excitation on device 1. As can be observed, different radiative transitions belonging to the excited state levels of $\mathrm{Er}^{3+}$ ions are detected when a DC voltage is applied. Note that the same line shape was always observed whatever the voltage (from $-25 \mathrm{~V}$ to $-45 \mathrm{~V})$. In particular, even the most energetic peaks appear at low voltages, then preserving a linear ratio between the EL of each peak and the current applied, evincing a direct excitation of $\mathrm{Er}^{3+}$ by means of hot carriers injected into the active layer [27].

On the contrary, under a symmetrical square wave voltage excitation (red line of figure 5), no $\mathrm{Er}^{3+}$ related peaks appear in the studied frequency range $(25 \mathrm{~Hz}-1 \mathrm{KHz})$ and solely a broad emission attributed to Si-ncs is observed. Furthermore, lower voltages are required to obtain luminescence in this regime, with a threshold voltage of $\pm 15 \mathrm{~V}$. Such value lays $10 \mathrm{~V}$ below the minimum DC threshold voltage observed in figure 2. 


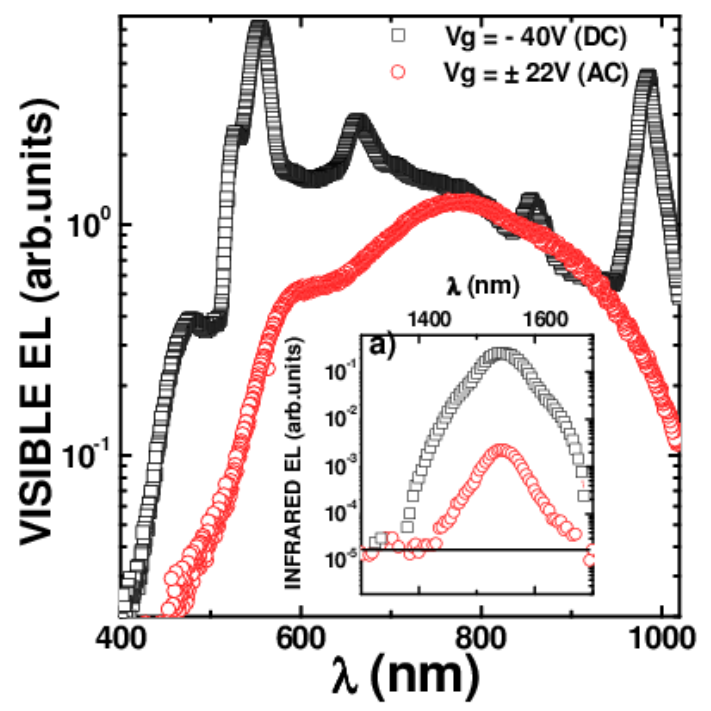

Figure 6: EL spectrum in the visible region for Device 1 under DC (black line) or symmetric AC (red line) polarization scheme. Infrared EL emission is shown in the inset.

Latest results provide evidences of different excitation mechanisms depending on the polarization applied in a single device. In particular, we are able to observe; (i) direct impact excitation of $\mathrm{Er}^{3+}$ ions under DC regime and (ii) energy transfer from $\mathrm{Si}$-ncs to $\mathrm{Er}^{3+}$ ions under symmetric square excitation. At this point, it is important to state that the applied APV voltage plays a principal role on the energy transfer: for values below the DC threshold $(<25 \mathrm{~V})$, carriers do not own enough energy to excite $\mathrm{Si}$-ncs or $\mathrm{Er}^{3+}$ ions directly, although exciton formation is allowed inside nanocrystals by sequential injection, resulting in the energy transfer to $\mathrm{Er}^{3+}$ ions. On the contrary, when symmetric APV regime reaches higher values than the threshold voltage in DC (>25V), a large number of carriers is able to excite by direct impact $\mathrm{Er}^{3+}$ (and also Sincs), screening almost completely the contribution of the energy transfer to the EL at $1.54 \mu \mathrm{m}$. In order to further support our interpretation, we did time-resolved measurements under APV excitation around the EL threshold voltage on device 1 (figure 2). Figure 7 shows the transition between the two different EL excitation mechanisms at $1.54 \mu \mathrm{m}$, depending on the APV voltage applied. As observed, a small shoulder starts to appear on the EL(t) signal at $\pm 25 \mathrm{~V}$ and it is reinforced as the voltage values become larger, suggesting the apparition of a contribution by direct impact in the excitation. Such shoulder completely screens the contribution of the indirect 
excitation at higher voltages, because i) the $\mathrm{Er}^{3+}$ ions excited by transfer are located in a region close to the Si substrate only and ii) the amount of $\mathrm{Er}^{3+}$ ions coupled to Si-nc is only a fraction of those who can be excited directly. Note finally that the transition voltage range (i.e. from energy transfer to impact ionization) is very narrow, about $2 \mathrm{~V}$ after the first signs of the EL shoulder.
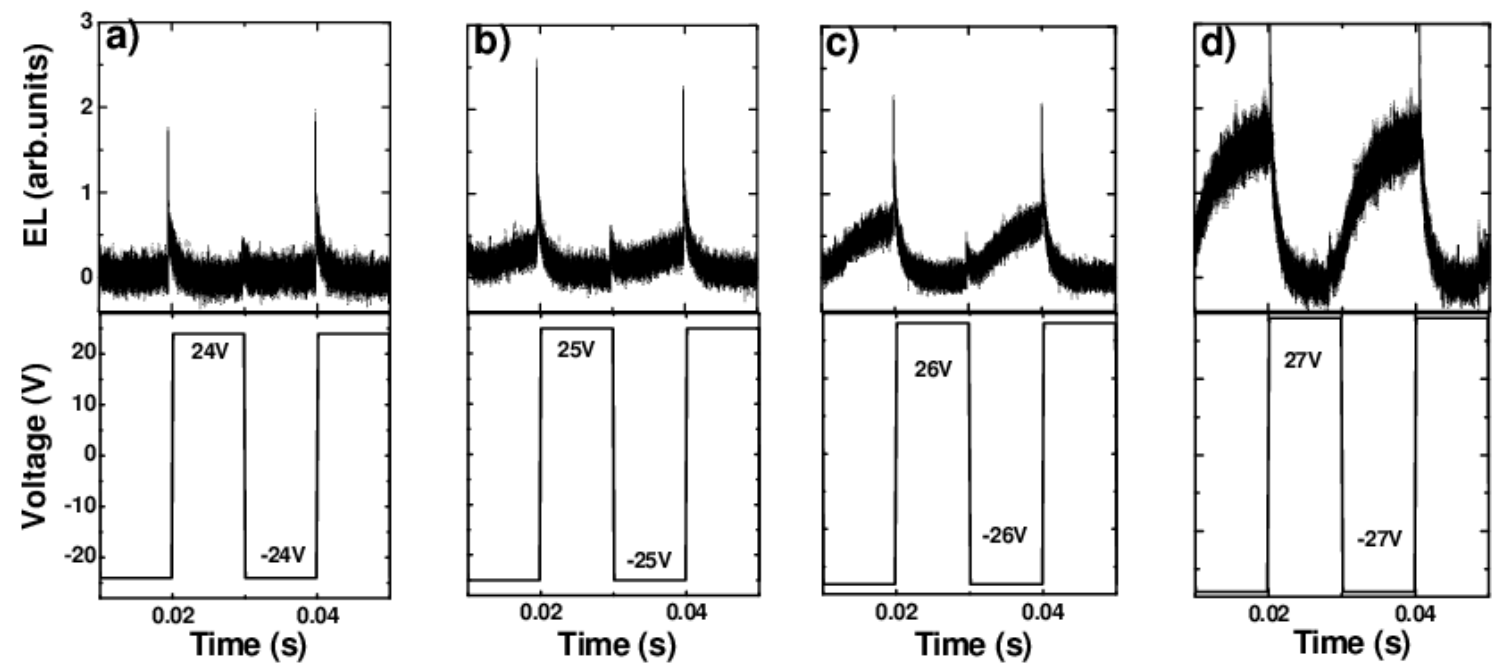

Figure 7: EL(t) at $1.54 \mu \mathrm{m}$ for different square symmetric voltage signals around the DC voltage threshold for device 1.

\subsection{Fast EL component}

In addition to the $\mathrm{Er}^{3+}$ ions $\mathrm{EL}$ dynamics at $1.54 \mu \mathrm{m}$ discussed above, at larger voltages sharp EL overshoots have been observed, characterized with much smaller decay time (in the order of few $\mu$ s). They could be observed either in samples with or without Si-ncs, as shown in figure 7 b,c,d for device 1 and figure 8 for device 2. In order to discard the influence of Si-nc, we have studied this feature on device 2 . As the device requires larger voltages, we had to reduce duty cycle to $10 \%$ in order to overcome the power limitation of our setup. This allowed us to observe solely one EL transient of typically $4 \mu$ s of decay time, as can be seen in figure 8 . Moreover, the same behavior was observed at $1300 \mathrm{~nm}$, which is away from any $\mathrm{Er}^{3+}$ related contribution, and also in the visible part of the spectrum (not shown). This fast component has already been 
observed by other authors $[26,28]$ when carrying out photoluminescence (PL) measurements in SRSO layers with and without $\mathrm{Er}^{3+}$ ions, obtaining very fast decay times (typically of nanoseconds). Nevertheless, the nature of this emission is not clear yet. Some authors $[28,29]$ suggest an effect of recombination of defective centers either in the silica matrix or at the interface with the Si-nc, or an intraband recombination mechanism [26]. Others attribute this fast emission to Auger processes related to transitions of confined electrons or holes between the space-quantized levels of Si-ncs [30]. In our case, it is clear that it is not provided by any $\mathrm{Er}^{3+}$ related transitions since it does not show its characteristic spectral features. Furthermore, this fast contribution is also appreciable in pure silica layers with $\mathrm{Er}^{3+}$ ions, suggesting that it may not be related to Si-ncs, but to defects on the silica matrix.

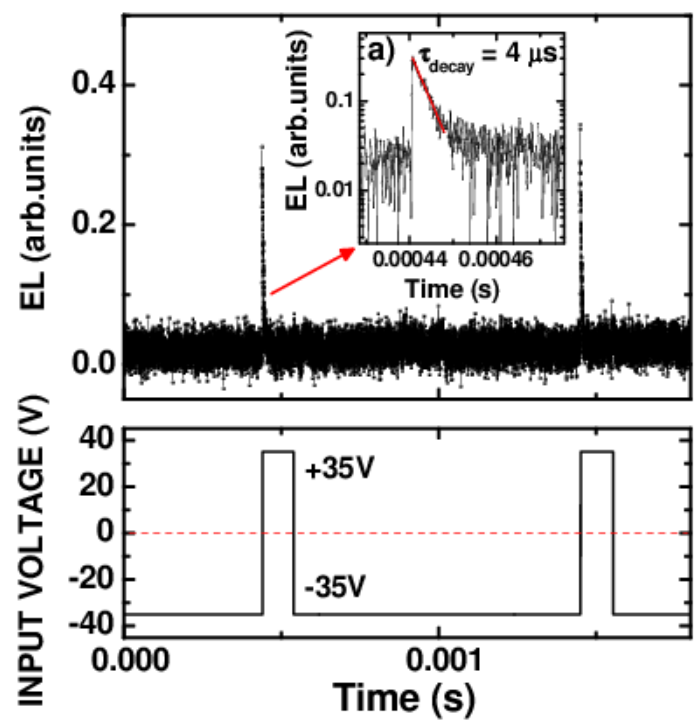

Figure 8: EL(t) at $1.54 \mu \mathrm{m}$ under a square symmetric voltage signal for device 2 . The inset shows the EL decay fit of the sharp EL overshoot.

In order to understand better the origin of the fast component, $\mu \mathrm{PL}$ measurements were accomplished. Active device area has been pumped at two different wavelengths, one resonant with an $\mathrm{Er}^{3+}$ transition $(488 \mathrm{~nm})$, and the other non-resonant $(476 \mathrm{~nm})$. The resultant $\mathrm{IR}$ emission was collected and integrated as a function of the photon flux in both devices, as observed in figure 9. The highest PL intensity in device 1 (inset of figure 9a) is obtained at 476 
$\mathrm{nm}$ (red circles), which is in strong agreement with an energy transfer mechanism from Si-ncs to $\mathrm{Er}^{3+}$ ions [31]. Indeed, the absorption cross-section of Si-ncs under $476 \mathrm{~nm}$ pumping wavelength is slightly higher than at $488 \mathrm{~nm}$. On the contrary, in the sample free of Si-ncs (device 2), it is the $488 \mathrm{~nm}$ pumping which gives higher PL intensity values (inset of figure 9b, black squares). Weak PL was observed under $476 \mathrm{~nm}$ pumping. Nonetheless, there is a small PL emission at $1.54 \mu \mathrm{m}$ from the non-resonant pumping in device 2 (see inset of figure $9 \mathrm{~b}$ ). This is a quite surprising result, since it seems that there is a small contribution from sensitized $\mathrm{Er}^{3+}$, whose origin is currently under study. Furthermore, a background emission was detected along a spectral range much broader than that covered by the $\mathrm{Er}^{3+}$ emission in both devices (insets of figure 9a and figure 9b). This emission might be correlated with the fast EL peak observed under EL measurements (see inset of figure 8), which also showed an extremely broad spectral contribution.
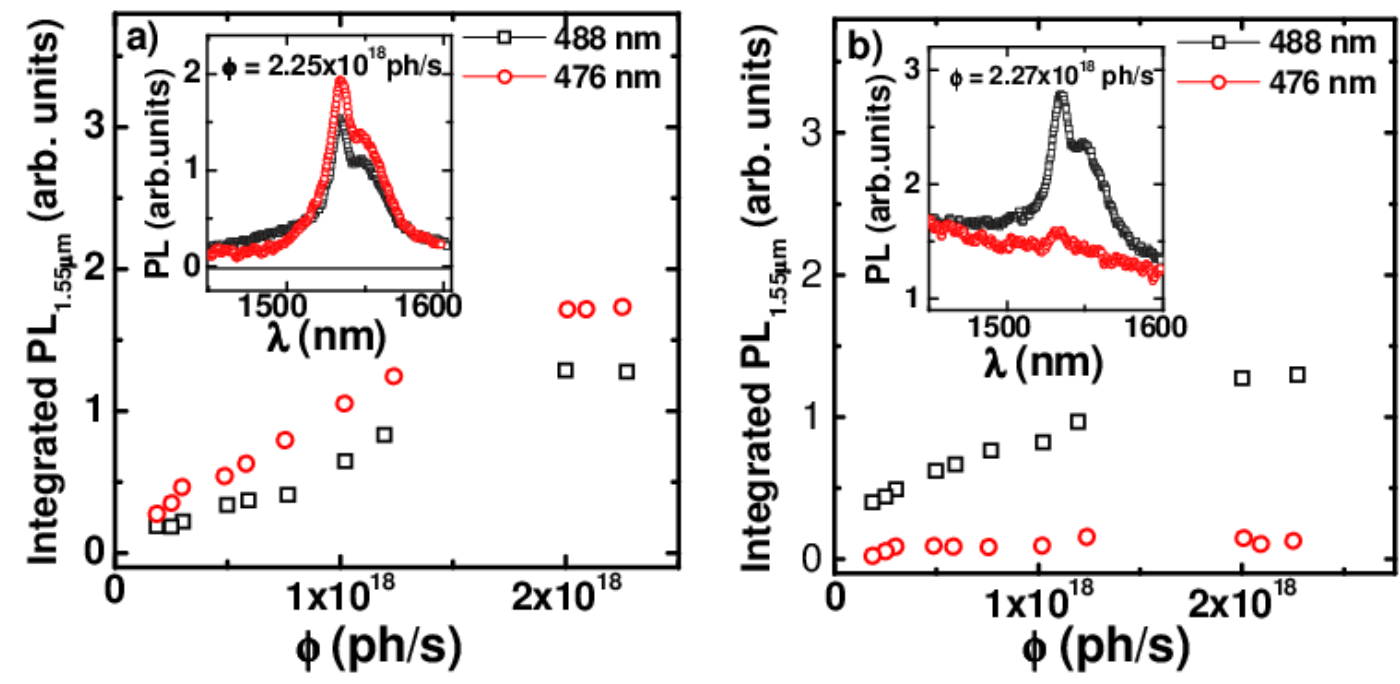

Figure 9: $\mu \mathrm{PL}$ intensity dependence with $\phi$ in device 1(a) and device 2(b). The insets show the obtained spectrum for a given $\phi$. The $y$ axis scales are comparable in both graphs.

\section{Conclusions}

In summary, we have studied the electroluminescence properties in the near infrared of silicon based LEDs containing silicon-rich silicon oxide layers doped with $\mathrm{Er}^{3+}$ ions under different 
polarization schemes. Time-resolved EL measurements allowed us to evidence the presence of two different excitation mechanism of $\mathrm{Er}^{3+}$ excitation under electrical pumping. Under DC excitation, Fowler-Nordheim injection and visible transition on the $\mathrm{Er}^{3+}$ spectra suggest that direct impact of $\mathrm{Er}^{3+}$ is the main mechanism. Under an alternate pulsed excitation, there is a window of voltages where transfer to $\mathrm{Er}^{3+}$ ions is clearly demonstrated, through the creation of exciton in the Si-nc by sequential injection of electrons and holes. Under this regime, a much smaller rise time is observed. This is a promising result, as this lead to a much larger absorption cross-section which can be used in an optimized material to obtain a much larger efficiency of pumping.

Finally, a fast EL overshoot observed in $\mathrm{Er}^{3+}: \mathrm{SRSO}$ and $\mathrm{Er}^{3+}: \mathrm{SiO}_{2}$ devices at high alternate pulsed voltages was ascribed to the emission of defects in the host silica matrix, and correlated with the background emission in $\mu \mathrm{PL}$ measurements.

\section{Acknowledgements}

This work was supported by EC through the project ICT-FP7-224312 HELIOS and by ItalySpain integrated actions.

\section{References}

[1] Jambois O, Gourbilleau F, Kenyon A J, Montserrat J, Rizk R and Garrido B 2010 Opt. Express. 182230

[2] Yerci S, Li R, Kucheyev S O, Van Buuren T, Basu S N and Dal Negro L 2009 Appl. Phys. Lett. 95031107

[3] Gallis S, Huang M, Efstathiadis H, Eisenbraun E, Kaloyeros A E, Nyein E E and Hommerich U 2005 Appl. Phys. Lett. 87091901

[4] Wodjak M, Klik M, Forcales M, Gusev O B, Gregorkiewicz T, Pacifici D, Franzò G, Priolo F, Iacona F 2004 Phys. Rev. B 69233315

[5] Sun K, Xu W J, Zhang B, You L P, Ran G Z and Qin G G 2008 Nanotechnology 19 105708 
[6] Miller G M, Briggs R M, Atwater H 2010 J. Appl. Phys. 108063109

[5] Shin J H, Lee W H and Han H S 1999 Appl. Phys. Lett 741573

[6] Izeddin I, Moskalenko A S, Yassievich I N, Fujii M and Gregorkiewicz T 2006 Phys. Rev. Lett 97207401

[7] Kik P G, Brongersma M L and Polman A 2000 Appl. Phys. Lett. 762325

[8] Navarro-Urrios D, Jambois O, Ferrarese Lupi F, Pellegrino P, Garrido B, Pitanti A, Prtljaga N, Daldosso N, Pavesi L 2011 Optical Materials 331086

[9] Ran G Z, Chen Y, Qin W C, Fu J S, Ma Z C, Zong W H, Lu H, Qin J and Qin G G $2001 \mathrm{~J}$. Appl. Phys. 905835

[10] Sun K, Xu W J, Zhang B, You L P, Ran G Z and Qin G G 2008 Nanotechnology 19 105708

[11] Sun J M, Skorupa W, Dekorsky T, Helm M and Nazarov A N 2005 Opt. Mater. 271050 [11] DiMaria D J, Dong D W, Falcony C, Theis T N, Kirtley J R, Tsang J C, Young D R, Pesavento F L and Brorson S D 1983 J. Appl. Phys. 545801

[12] Perálvarez M, Barreto J, Carreras J, Morales A, Navarro-Urrios D, Lebour Y, Domínguez C and Garrido B 2009 Nanotechnology 20405201

[13] Carreras J, Arbiol J, Garrido B, Bonafos C and Montserrat 2008 J. Appl. Phys. Lett 92 091103.

[14] Prtljaga N, Navarro-Urrios D, Marconi A, Anopchenko A, Colonna J P, Milesi F, Daldosso N, Jambois O, Garrido B, Fedeli J M, Pavesi L 2011 Opt. Materials 331083.

[15] DiMaria D J, Dong D W, Falcony C, Theis T N, Kirtley J R, Tsang J C, Young D R, and Pesavento F L 1983 J. Appl. Phys. 5410.

[16] Jambois O, Berencén Y, Hijazi K, Wodjak M, Kenyon A J, Gourbilleau F, Rizk R, Garrido B J. Appl. Phys. 200910663526.

[17] Konig D, Rennau M and Henker M 2007 Solid-State Electronics 51650

[18] Hadjadj A, Simonetti O, Maurel T, Salace G and Petit C 2002 Appl. Phys. Lett. 8018

[19] Lenzlinger M and Snow E H 1969 J. Appl. Phys. 401 
[20] DiMaria D J, Kirtley J R, Pakulis E J, Dong D W, Kuan T S, Pesavento F L, Theis T N and Cutro J A 1984 J. Appl. Phys. 562

[21] Coffa S, Franzò G and Priolo F 1996 Appl. Phys. Lett. 692077

[22] Wang S, Eckau A, Neufeld E, Carius R, and Buchal Ch 1997 Appl. Phys. Lett. 712824

[23] Lombardo S, Campisano S U, Van den Hoven G N and Polman A 1995 J. Appl. Phys. 77 6504

[24] Iacona F, Pacifici D, Irrera A, Miritello M, Franzò G, and Priolo F 2002 Appl. Phys. Lett. 813242

[25] Walters R J, Bourianoff G I and Atwater H A 2005 Nat. Mater. 4143

[26] Navarro-Urrios D, Pitanti A, Daldosso N, Gourbilleau F, Rizk R, Garrido B, and Pavesi L 2009 Phys. Rev. B 79193312

[27] Kanjilal A, Rebohle L, Skorupa W and Helm M 2009 Appl. Phys. Lett 94101916

[28] Izeddin I, Timmerman D, Gregorkiewicz T, Moskalenko A S, Prokofiev A A and Yassievich I N 2008 Phys. Rev. B. 780.35327

[29] Savchyn O, Ruhge F R, Kik P G, Todi R M, Coffey K R, Nukala H and Heinrich H 2007 Phys. Rev. B, 76195419

[30] Prokofiev A A, Moskalenko A S, Yassievich I N 2008 Materials Science and Engineering B 146121

[31] Fujii M, Yoshida M, Kanzawa Y, Hayashi S and Yamamoto K 1997 Appl. Phys. Lett. 71 1198 
